\title{
Immune stimulation reduces sleep and memory ability in Drosophila melanogaster
}

Psychoneuroimmunology studies the increasing number of connections between neurobiology, immunology and behaviour. We demonstrate the effects of the immune response on two fundamental behaviours: sleep and memory ability in Drosophila melanogaster. We used the Geneswitch system to upregulate peptidoglycan receptor protein (PGRP) expression, thereby stimulating the immune system in the absence of infection. Geneswitch was activated by feeding the steroid RU486, to the flies. We used an aversive classical conditioning paradigm to quantify memory and measures of activity to infer sleep. Immune stimulated flies exhibited reduced levels of sleep, which could not be explained by a generalised increase in waking activity. Immune stimulated flies also showed a reduction in memory abilities. These results lend support to Drosophila as a model for immune-neural interactions and provide a possible role for sleep in the interplay between the immune response and memory. 
$3{ }^{a}$ Biology Department, University of Leicester, Leicester LE1 7RH, United Kingdom

$4{ }^{\mathrm{b}}$ Department of Biology, Taif university, Saudi Arabia

$5 \quad{ }^{\mathrm{c}}$ Genetics Department, University of Leicester, Leicester LE1 7RH, United Kingdom

6 Corresponding author

7 Eamonn Mallon

8 Department of Biology

$9 \quad$ University of Leicester

10 Tel: $+44(0) 1162523488$

11 Fax: $+44(0) 1162523330$

12 Email: ebm3@1e.ac.uk 
Introduction

14 Psychoneuroimmunology, in vertebrates, studies the connections between neurobiology, 15 immunology and behaviour (Ader, Felten, \& Cohen, 1991). These neural-immune interactions 16 have also been found in invertebrates (Demas, Adamo, \& French, 2011). For example, immune 17 response negatively affects learning and memory in bees (Mallon, Brockmann, \& Schmid18 Hempel, 2003; Gegear, Otterstatter, \& Thomson, 2006; Riddell \& Mallon, 2006; Iqbal \& Mueller, 19 2007; Alghamdi, Dalton, Phillis, Rosato, \& Mallon, E.B., 2008). A tractable invertebrate model of these immune-neural links would provide a stimulus to this field (Aubert, 2007). The fruit fly, Drosophila melanogaster, has been tremendously helpful to the analysis of associative learning (Kim, Lee, \& Han, 2007) and immunity (Lemaitre \& Hoffmann, 2007). In this paper we demonstrate immune-memory links in Drosophila and further expand the paradigm by showing immune-sleep interactions in flies.

Sleep is a resting state where the sleeper exhibits inattention to the environment and is usually immobile (Siegel, 2003). Drosophila melanogaster like vertebrates have been shown to have a distinct sleep state. In flies, a sleep episode is defined as a period of immobility lasting five minutes or longer (Hendricks, Finn, Panckeri, Chavkin, Williams, Sehgal, \& Pack, 2000; Shaw, Cirelli, Greenspan, \& Tononi, 2000). Such intervals are associated with reversible increases in arousal threshold, which can be further augmented following sleep deprivation (Huber, Ghilardi, Massimini, \& Tononi, 2004), are associated with changes in brain electrical activity (Nitz, Van Swinderen, Tononi, \& Greenspan, 2002; Alphen, Yap, Kirszenblat, Kottler, \& Swinderen, 2013), and are reduced by several drugs like caffeine and modafinil and are increased by antihistamines

34 (Hendricks, Finn, Panckeri, Chavkin, Williams, Sehgal, \& Pack, 2000; Shaw, Cirelli, Greenspan,

$35 \&$ Tononi, 2000). As in mammals, sleep deprivation leads to a rebound in quantity of sleep 36 (Shaw, Cirelli, Greenspan, \& Tononi, 2000). 
37 Infections increase sleep in humans, most likely through induction of proinflammatory cytokines

38 (Bryant, Trinder, \& Curtis, 2004). Fruit flies infected with gram-negative bacteria also show 39 increased sleep (Kuo, Pike, Beizaeipour, \& Williams, 2010). Another study found flies infected 40 with gram-positive bacteria slept less (Shirasu-Hiza, Dionne, Pham, Ayres, \& Schneider, 2007).

41 Here, we activated the immune system non-pathogenically (Moret \& Schmid-Hempel, 2000; 42 Mallon, Brockmann, \& Schmid-Hempel, 2003; Riddell \& Mallon, 2006; Alghamdi, Dalton, 43 Phillis, Rosato, \& Mallon, E.B., 2008; Richard, Aubert, \& Grozinger, 2008). This separates the 44 effect of the immune response from any direct effect of the pathogen, for example, parasite 45 manipulation of the host (Adamo \& Webster, 2013). We used Geneswitch (Osterwalder, Yoon, 46 White, \& Keshishian, 2001) to up-regulate peptidoglycan receptor protein LCa (PGRP-Lca) in 47 adult flies. PGRP-Lca is a pattern recognition protein that recognizes DAP type peptidoglycan 48 which is found on Gram negative and Gram positive bacteria setting off the IMD immune 49 pathway and leading to the expression of antimicrobial peptides (Gottar, Gobert, Michel, Belvin, 50 Duyk, Hoffmann, Ferrandon, \& Royet, 2002). Geneswitch is activated in the presence of the 51 steroid RU486. We used an aversive classical conditioning paradigm to measure memory abilities 52 of flies (Mery \& Kawecki, 2005). Sleep was measured using the Drosophila Activity Monitoring 53 System 2 (DAMS2, Trikinetics, Waltham, MA). 
Methods and Materials

55 The Geneswitch line $w^{1118} ; \mathrm{P}\left\{\mathrm{w}^{+\mathrm{mW} . h s}=\right.$ Switch 1$\}$ bun ${ }^{\text {Switchl.32 }}$ (hereafter referred to as GS1.32) drives 56 expression of RU486-activated GAL4 in adult fat bodies (Gottar, Gobert, Michel, Belvin, Duyk, 57 Hoffmann, Ferrandon, \& Royet, 2002) (http://flystocks.bio.indiana.edu). The three genotypes used were GS1.32>PGRP-Lca( $w^{1118} ; G S 1.32 /+$; UAS-PGRP-Lca/+), and the control genotypes GS1.32/+(w ${ }^{1118} ;$ GS1.32/+; +/+) and +/PGRP-Lca (+/+;UAS-PGRP-Lca/+).

Flies were maintained in vials containing agar, sugar, and Brewer's yeast media in a $12 \mathrm{~h}: 12 \mathrm{~h}$

61 light: dark cycle at $25^{\circ} \mathrm{C}$. This food was also used during all behavioural assays. Males and females were selected at eclosion and flies were 1-3 days old at the beginning of the experiment. Both sexes were used for the memory assay and sleep assay (Isaac, Li, Leedale, \& Shirras, 2010).

64

\section{Geneswitch}

In the Geneswitch system, the DNA binding domain of the GAL4 protein is fused to the activation moiety of p65 through a mutant progesterone receptor ligand binding domain. Thus, Geneswitch is a chimeric ligand-stimulated activator of transcription. In the absence of ligand, the Geneswitch is in the "off" state. In the presence of the antiprogestin RU486 the Geneswitch molecule changes to an active conformation, in which it binds, as a dimer, to UAS sequences and activates transcription of downstream genes. In flies, Geneswitch mediated expression can be detectable 3-5 hr after feeding on RU486, reaching maximal levels 21-48 hr later (Osterwalder, Yoon, White, \& Keshishian, 2001; Roman, Endo, Zong, \& Davis, 2001).

$20 \mathrm{ml}$ of RU486 (Sigma Aldrich) $10 \mathrm{mM}$ stock solution $(0.13 \mathrm{~g}$ of RU486 in $32 \mathrm{ml}$ of $80 \%$ ethanol) was mixed with $980 \mathrm{ml}$ molten Drosophila food (200 $\mu \mathrm{M}$ final concentration). For the 
75 memory assay, flies were fed for two days with RU486 before the start of the training and

76 returned to the RU486 food after training. For the sleep assay, flies were placed in vials

77 containing RU486 food for two days to allow feeding. After two days flies were immediately

78 loaded into tubes containing more of the RU486 food. For all lines we have flies fed with RU486

79 and genetically identical animals cultured on fly medium supplemented with an equal amount of 80 vehicle (80\% ethanol) that lacked RU486.

\section{Memory assay}

82 Each sample was a single sex group of 50 adult flies. This memory assay was described 83 previously (Mery \& Kawecki, 2005). Conditioning consisted of 5 training sessions separated by

$8420 \mathrm{~min}$ intervals. In each training session flies were first exposed for $30 \mathrm{~s}$ to one odorant 85 simultaneously with mechanical shock delivered every $5 \mathrm{~s}$. This period was followed by a $60 \mathrm{~s}$ rest 86 period (no odour and no shock). Then, for 30s another odorant was delivered, without shock.

87 Flies were either conditioned against 3-octanol or 4-methylcyclohexanol (both $0.6 \mathrm{ml} / 1$ of 88 paraffin).

8924 hours after the conditioning period flies were transported to the choice point of a T-maze,

90 where they were allowed to choose between the two odors for 60 s. The memory score was the 91 proportion of individuals choosing the correct odour, i.e. not the one they were trained against.

92 One hundred and fifteen replicates were carried out, distributed between the genotype, sex, 93 RU486 (presence/absence) and odour used. 
94

95

96

97

98

99

$100 \quad 28.75$ flies
$101 \quad$ Data analys

$100 \quad 28.75$ flies
$101 \quad$ Data analys

102

103

104

105

106

107

108

109

110

111

112

113

114 the control genotypes. Using a Bonferroni correction the significance level $\alpha$ was reduced to

$1150.0033(0.05 / 15)$ for the sleep data, as there were three separate ANOVAs carried out for each of

1165 measures. All analysis was carried out using R 3.01 (R Core Team, 2013). 


\section{Zone of inhibition assay}

118 Our treatment line had previously been shown to upregulate the immune response (Gottar et al. 119 2002). However we used the zone of inhition assay to confirm increased immune response in our 120 treated flies. This assay measures antibacterial activity: it is based on the ability of immune 121 proteins to inhibit bacterial growth when placed onto an agar plate seeded with bacteria 122 (Arthrobacteur globiformis $125 \mu \mathrm{l}$ of an overnight culture per 50ml of agar). Thirty seven 123 GS1.32>PGRP-Lca flies, 17 fed RU486 and 20 not fed RU486 were used. Each fly was 124 homogenized in $30 \mu \mathrm{l}$ of ringer solution. Five microlitres of the supernatant from the centrifuged 125 solution $\left(1300 \mathrm{~g}\right.$ for $10 \mathrm{~min}$ at $\left.4^{\circ} \mathrm{C}\right)$ were pippetted into a hole on the agar plate. This was 126 incubated for $48 \mathrm{hrs}\left(30^{\circ} \mathrm{C}\right)$. The resultant ZOI were measured as the mean of three diameters. 
128 Raw data is available on figshare (http://dx.doi.org/10.6084/m9.figshare.1030499). Feeding RU486 to GS1.32>PGRP-Lca flies increased their antibacterial activity by $26 \%$ (mean+/130 standard error: RU486 $+=5.85 \mathrm{~mm}+/-0.25$, RU486- $=4.65+/-0.45, \mathrm{t}=-2.3263, \mathrm{df}=29.202, \mathrm{p}=$ $1310.02715)$

132 Immune stimulation effects on memory

133 Genotype had a significant effect on memory score $\left(F_{2,109}=22.46, p<0.0001\right)$. The main effects 134 for sex, whether RU486 was used, and odour used were not significant. There was however a 135 signifcant interaction between genotype and whether RU486 was used $\left(\mathrm{F}_{2,109}=5.76, \mathrm{p}=0.0042\right)$. 136 GS1.32>PGRP-Lca flies, showed a 11.4\% decrease in memory scores when fed RU468 relative 137 to those not fed RU468 of the same genotype (Tukey HSD: GS1.32>PGRP-Lca + RU468 versus 138 GS1.32>PGRP-Lca - RU468 $\mathrm{p}=0.0418$, GS1.32/ + RU486 versus GS1.32/ + - RU486 $\mathrm{p}=$ 139 0.9578, +/PGRP-Lca + RU486 versus +/PGRP-Lca - RU486 $\mathrm{p}==0.6784)$. See Figure 1. As 140 feeding RU486 to GS1.32>PGRP-Lca flies leads to an increased immune response, immune 141 stimulation decreases memory scores.

142 Immune stimulation effects on sleep

143 The complete ANOVAs for all measures are available in the supplementary materials. Immune 144 stimulated flies (GS1.32>PGRP-Lca fed with RU486) showed a decrease in sleep relative to 145 controls (Complete 4-way ANOVA genotype:ru $\mathrm{F}_{2,23976}=158.74, \mathrm{p}<0.00001$, GS1.32>PGRP146 Lca vs GS1.32/+: $F_{1,16632}=282.37, \mathrm{p}<0.00001$, GS1.32 $>$ PGRP-Lca vs +/ PGRP-Lca: $\mathrm{F}_{1,15840}=$ 147 11.82, $\mathrm{p}=0.00059)$. See Figure 2 and Figure 3. Table 1 gives the means and standard errors. As 148 there was no significant interaction between sex, genotype and whether RU486 was used 149 (Complete 4-way ANOVA genotype:ru $\mathrm{F}_{2,23976}=20.79, \mathrm{p}<0.00001$, GS1.32>PGRP-Lca vs 
GS1.32/+: $\mathrm{F}_{1,16632}=32.75, \mathrm{p}<0.00001, \mathrm{GS} 1.32>$ PGRP-Lca vs $+/$ PGRP-Lca: $\mathrm{F}_{1,15840}=0.01, \mathrm{p}=$

151

152

153

154

155

156

157

158

159

160

161

162

163

164

165

166

167

168

169

0.90374) a single sex analysis is not shown. However dividing into males and females did not alter the significant effect of immune response on sleep.

Immune response had no effect on mean waking activity (Complete 4-way ANOVA genotype:ru $\mathrm{F}_{2,23976}=21.96, \mathrm{p}<0.00001, \mathrm{GS} 1.32>$ PGRP-Lca vs GS1.32/+: $\mathrm{F}_{1,16632}=0.49, \mathrm{p}=0.4858$ GS1.32>PGRP-Lca vs +/ PGRP-Lca: $\left.\mathrm{F}_{1,15840}=39.18, \mathrm{p}<0.00001\right)$. See Figure 4 and Figure 5. Table 2 gives the means and standard errors for mean waking activity.

Immune response had a significant effect on number of sleep bouts (Complete 4-way ANOVA genotype:ru $\mathrm{F}_{2,23976}=69.9, \mathrm{p}<0.00001, \mathrm{GS1} 1.32>$ PGRP-Lca vs GS1.32/+: $\mathrm{F}_{1,16632}=013.08, \mathrm{p}=$ 0.0003, GS1.32>PGRP-Lca vs +/ PGRP-Lca: $\left.F_{1,15840}=71.52, p<0.00001\right)$. See Figure 6 and Figure 7. Table 3 gives the means and standard errors for number of sleep bouts. This result is difficult to interpret as RU486+ GS1.32>PGRP-Lca flies have more disturbed sleep than RU486+ GS1.32/+ flies but less disturbed sleep than RU486+ +/ PGRP-Lca flies.

Immune response had no effect on sleep bout duration (Complete 4-way ANOVA genotype:ru $\mathrm{F}_{2,23976}=203.49, \mathrm{p}<0.00001$, GS1.32 $>$ PGRP-Lca vs GS1.32/+: $\mathrm{F}_{1,16632}=290.48, \mathrm{p}<0.00001$, GS1.32>PGRP-Lca vs +/ PGRP-Lca: $\mathrm{F}_{1,15840}=7.78, \mathrm{p}=0.0053$ (Not significant)). See Figure 8 and Figure 9. Table 4 gives the means and standard errors for sleep bout duration.

Immune response had no effect on sleep latency (Complete 4-way ANOVA genotype:ru $\mathrm{F}_{2,925}=$ $1.78, \mathrm{p}=0.16935$ ). See Figure 10 and Figure 11. Table 5 gives the means and standard errors for sleep latency. 
Discussion

171 Immune stimulated adult flies exhibit reduced levels of sleep both during day and night. Immune

172 stimulation also leads to a reduction in memory abilities.

173 The reduction in sleep cannot be explained simply in terms of a generalised increase in activity.

174 Stimulating the immune response had no effect on mean waking activity during the day or night,

175 but immune-stimulated flies slept less than the non-stimulated controls.

176 As we did not test for the effects of immune response on olfactory sensitivity itself, we can not 177 say categorically that our effect on memory was not due to a decrease in the flies' ability to 178 differentiate odours. However, experiments carried out on honeybees (Mallon, Brockmann, \& 179 Schmid-Hempel, 2003) and bumblebees (Riddell \& Mallon, 2006) found that the immune 180 response did not effect their olfaction.

Our sleep results are difficult to relate to previous sttudies that found an effect of infection on sleep in Drosophila. Shirasu-Hiza showed a decrease in sleep after gram-positive bacterial infections (Shirasu-Hiza, Dionne, Pham, Ayres, \& Schneider, 2007). However Kuo (Kuo, Pike, Beizaeipour, \& Williams, 2010) found that when they infected flies with gram-negative bacteria, the flies slept more. These two results are difficult to compare as the experiments differed in numerous methodical aspects, e.g. strength of infection, lighting paradigm, when the phenotype was measured, etc.

Although Imd is one of the canonical immune pathways in insects, over-expression of the Imd pathway can also lead to apoptosis (Georgel, Naitza, Kappler, Ferrandon, Zachary, Swimmer, Kopczynski, Duyk, Reichart, \& Hoffmann, 2001; Leulier, Parquet, Pili-Floury, Ryu, Caroff, Lee, 191 Mengin-Lecreulx, \& Lemaitre, 2003). It cannot be excluded that our results could be caused by a 
192 side effect: apoptosis of the fat body by the Imd pathway rather than its main effect of immune 193 response. This will be examined in future work.

194 We have shown that immune response decreases sleep and memory in Drosophila melanogaster.

195 We propose a possible link between all three systems as an interesting area for future research.

196 One of the main hypotheses on sleep function is that sleep periods are favourable for brain 197 plasticity and in the adult brain for learning and memory (Maquet, 2001). Like humans, flies with 198 a fragmented sleep show impaired learning compared with flies with consolidated sleep (Seugnet, 199 Suzuki, Vine, Gottschalk, \& Shaw, 2008). Flies also exhibit decreases in learning after 6 or 12 200 hours of sleep deprivation (Seugnet, Suzuki, Vine, Gottschalk, \& Shaw, 2008). We propose sleep 201 as an intermediate between immunity and memory. We hypothesise that it is not the activation of 202 the immune system per se that affects memory in flies, but rather that immune stimulation 203 reduces the length and quality of sleep that in turn, reduces memory ability. However, with our 204 current data, we cannot exclude that in flies the level of immune activation has a direct effect on 205 memory.

206 Our results adds to the evidence for Drosophila as a model for immune-neural interactions. As 207 well as the potential use as a model for mammalian neural-immune links, this work has direct 208 impact on recent concern for insect foragers and the role of multiple stressors in their decline 209 (Gill, Ramos-Rodriguez, \& Raine, 2012). 
211 Thanks to Dr. Frederic Mery (CNRS, Gif Sur Yvette) for initial discussions about setting up the

212 memory assay. Thanks to E. Green (Genetics, University of Leicester) for use of the excel plugin,

213 Befly to calculate sleep measures. 
References

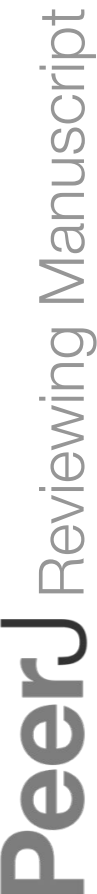

PeerJ reviewing PDF | (v2014:03:1684:1:0:NEW 20 May 2014) 
Adamo, S. A., \& Webster, J. P. 2013. Neural parasitology: how parasites manipulate host behaviour. The Journal of Experimental Biology 216(1):1-2.

214 Ader, R., Felten, D. L., \& Cohen, N. 1991. Psychoneuroimmunology (2nd ed.). San Diego: 215 Academic Press.

216 Alghamdi, A., Dalton, L., Phillis, A., Rosato, E., \& Mallon, E.B. 2008. Immune response impairs 217 learning in free-flying bumble-bees. Biology Letters 4:479-481.

218

219

220
Alphen, B. van, Yap, M. H. W., Kirszenblat, L., Kottler, B., \& Swinderen, B. van. 2013. A Dynamic Deep Sleep Stage in Drosophila. The Journal of Neuroscience 33(16):6917-6927.

Aubert, A. 2007. Invertebrate studies and the evolution of comparative psychoneuroimmunology. Brain Behavior and Immunity 21:290-291.

Bryant, P. A., Trinder, J., \& Curtis, N. 2004. Sick and tired: Does sleep have a vital role in the immune system? Nature Reviews Immunology 4:457-467.

Demas, G. E., Adamo, S. A., \& French, S. S. 2011. Neuroendocrine-immune crosstalk in vertebrates and invertebrates: implications for host defence. Functional Ecology 25:29-39.

Gegear, R. J., Otterstatter, M. C., \& Thomson, J. D. 2006. Bumble-bee foragers infected by a gut parasite have an impaired ability to utilize floral information. Proceedings of the Royal Society B-Biological Sciences 273:1073-1078.

Georgel, P., Naitza, S., Kappler, C., Ferrandon, D., Zachary, D., Swimmer, C., Kopczynski, C., Duyk, G., Reichart, J. M., \& Hoffmann, J. A. 2001. Drosophila immune deficiency (IMD) is a death domain protein that activates antibacterial defense and can promote apoptosis. Developmental Cell 1:503-514.

Gill, R. J., Ramos-Rodriguez, O., \& Raine, N. E. 2012. Combined pesticide exposure severely affects individual-and colony-level traits in bees. Nature Genetics 491:105-108.

Gottar, M., Gobert, V., Michel, T., Belvin, M., Duyk, G., Hoffmann, J. A., Ferrandon, D., \& Royet, J. 2002. The Drosophila immune response against Gram-negative bacteria is mediated by a peptidoglycan recognition protein. Nature 416:640-644.

Hendricks, J. C., Finn, S. M., Panckeri, K. A., Chavkin, J., Williams, J. A., Sehgal, A., \& Pack, A. I. 2000. Rest in Drosophila is a sleep-like state. Neuron 25:129-138.

Huber, R., Ghilardi, M. F., Massimini, M., \& Tononi, G. 2004. Local sleep and learning. Nature 430:78-81.

Iqbal, J., \& Mueller, U. 2007. Virus infection causes specific learning deficits in honeybee foragers. Proceedings of the Royal Society B-Biological Sciences 274:1517-1521.

Isaac, R. E., Li, C. X., Leedale, A. E., \& Shirras, A. D. 2010. Drosophila male sex peptide inhibits siesta sleep and promotes locomotor activity in the post-mated female. Proceedings of the Royal Society B-Biological Sciences 277:65-70. 
247 Kim, Y. C., Lee, H. G., \& Han, K. A. 2007. Classical reward conditioning in Drosophila 248 melanogaster. Genes Brain and Behavior 6:201-207.

249 Kuo, T. H., Pike, D. H., Beizaeipour, Z., \& Williams, J. A. 2010. Sleep triggered by an immune 250 response in Drosophila is regulated by the circadian clock and requires the NF kappa B Relish. 251 Bmc Neuroscience 11(17).

252 Lemaitre, B., \& Hoffmann, J. 2007. The host defense of Drosophila melanogaster. Annual 253 Review of Immunology 25:697-743.

254 Leulier, F., Parquet, C., Pili-Floury, S., Ryu, J. H., Caroff, M., Lee, W. J., Mengin-Lecreulx, D., \& 255 Lemaitre, B. 2003. The Drosophila immune system detects bacteria through specific 256 peptidoglycan recognition. Nature Immunology 4:478-484.

Mallon, E. B., Brockmann, A., \& Schmid-Hempel, P. 2003. Immune response inhibits associative 258 learning in insects. Proceedings of the Royal Society of London Series B-Biological Sciences 259 270:2471-2473.

260 Maquet, P. 2001. The role of sleep in learning and memory. Science 294:1048-1052.

Mery, F., \& Kawecki, T. J. 2005. A cost of long-term memory in Drosophila. Science 308:1148262

Moret, Y., \& Schmid-Hempel, P. 2000. Survival for immunity: The price of immune system 264

Nitz, D. A., Van Swinderen, B., Tononi, G., \& Greenspan, R. J. 2002. Electrophysiological correlates of rest and activity in Drosophila melanogaster. Current Biology 12:1934-1940.

Osterwalder, T., Yoon, K. S., White, B. H., \& Keshishian, H. 2001. A conditional tissue-specific transgene expression system using inducible GAL4. Proceedings of the National Academy of Sciences of the United States of America 98:12596-12601.

R Core Team. 2013. $R$ : A language and environment for statistical computing. Vienna, Austria: $\mathrm{R}$ foundation for statistical computing.

Richard, F.-J., Aubert, A., \& Grozinger, C. M. 2008. Modulation of social interactions by immune stimulation in honey bee, Apis mellifera, workers. BMC Biology 6(1):50.

Riddell, C. E., \& Mallon, E. B. 2006. Insect psychoneuroimmunology: Immune response reduces learning in protein starved bumblebees (Bombus terrestris). Brain, Behavior and Immunity 20:135-138.

Roman, G., Endo, K., Zong, L., \& Davis, R. L. 2001. P (Switch), a system for spatial and temporal control of gene expression in Drosophila melanogaster. Proceedings of the National Academy of Sciences 98(22):12602-12607.

Seugnet, L., Suzuki, Y., Vine, L., Gottschalk, L., \& Shaw, P. J. 2008. D1 Receptor Activation in the Mushroom Bodies Rescues Sleep-Loss-Induced Learning Impairments in Drosophila. Current Biology 18(15):1110-1117. 
283 Shaw, P. J., Cirelli, C., Greenspan, R. J., \& Tononi, G. 2000. Correlates of sleep and waking in 284 Drosophila melanogaster. Science 287:1834-1837.

285 Shirasu-Hiza, M. M., Dionne, M. S., Pham, L. N., Ayres, J. S., \& Schneider, D. S. 2007. 286 Interactions between circadian rhythm and immunity in Drosophila melanlogaster. Current 287 Biology 17:R353-R355.

288 Siegel, J. M. 2003. Why we sleep. Scientific American 289:92-97. 
Figure 1. Memory score for each genotype. Memory score is the proportion of flies that choose the odour they were not trained against. The blue boxes represent the mean memory score for the RU486- flies. The red boxes represent the RU486+ flies. The grey dots are the individual data points.

Figure 2. Sleepbins for the males for each genotype. The blue points represent the means for the RU486- flies. The red points represent the means of RU486+ flies. Error bars are standard errors. The shaded times are night (lights off).

Figure 3. Sleepbins for the females for each genotype. The blue points represent the means for the RU486- flies. The red points represent the means of RU486+ flies. Error bars are standard errors. The shaded times are night (lights off).

Figure 4. Mean waking activity for the males for each genotype. The blue points represent the means for the RU486- flies. The red points represent the means of RU486+ flies. Error bars are standard errors. The shaded times are night (lights off).

Figure 5. Mean waking activity for the females for each genotype. The blue points represent the means for the RU486- flies. The red points represent the means of RU486+ flies. Error bars are standard errors. The shaded times are night (lights off).

Figure 6. Number of sleep bouts per hour for the males for each genotype. The blue points represent the means for the RU486- flies. The red points represent the means of RU486+ flies. Error bars are standard errors. The shaded times are night (lights off).

Figure 7. Number of sleep bouts per hour for the females for each genotype. The blue points represent the means for the RU486- flies. The red points represent the means of RU486+ flies. Error bars are standard errors. The shaded times are night (lights off).

Figure 8. Sleep bout duration for the males for each genotype. The blue points represent the means for the RU486- flies. The red points represent the means of RU486+ flies. Error bars are standard errors. The shaded times are night (lights off).

Figure 9. Sleep bout duration for the females for each genotype. The blue points represent the means for the RU486- flies. The red points represent the means of RU486+ flies. Error bars are standard errors. The shaded times are night (lights off).

Figure 10. Sleep latency for the males for each genotype. The blue points represent the means for the RU486- flies. The red points represent the means of RU486+ flies. Error bars are standard errors.

Figure 11. Sleep latency for the females for each genotype. The blue points represent the means for the RU486- flies. The red points represent the means of RU486+ flies. Error bars are standard errors. 


\section{Figure 1}

Memory score for each geneotype

Fig 1. Memory score for each genotype. Memory score is the proportion of flies that choose the odour they were not trained against. The blue boxes represent the mean memory score for the RU486- flies. The red boxes represent the RU486+ flies. The grey dots are the individual data points. 


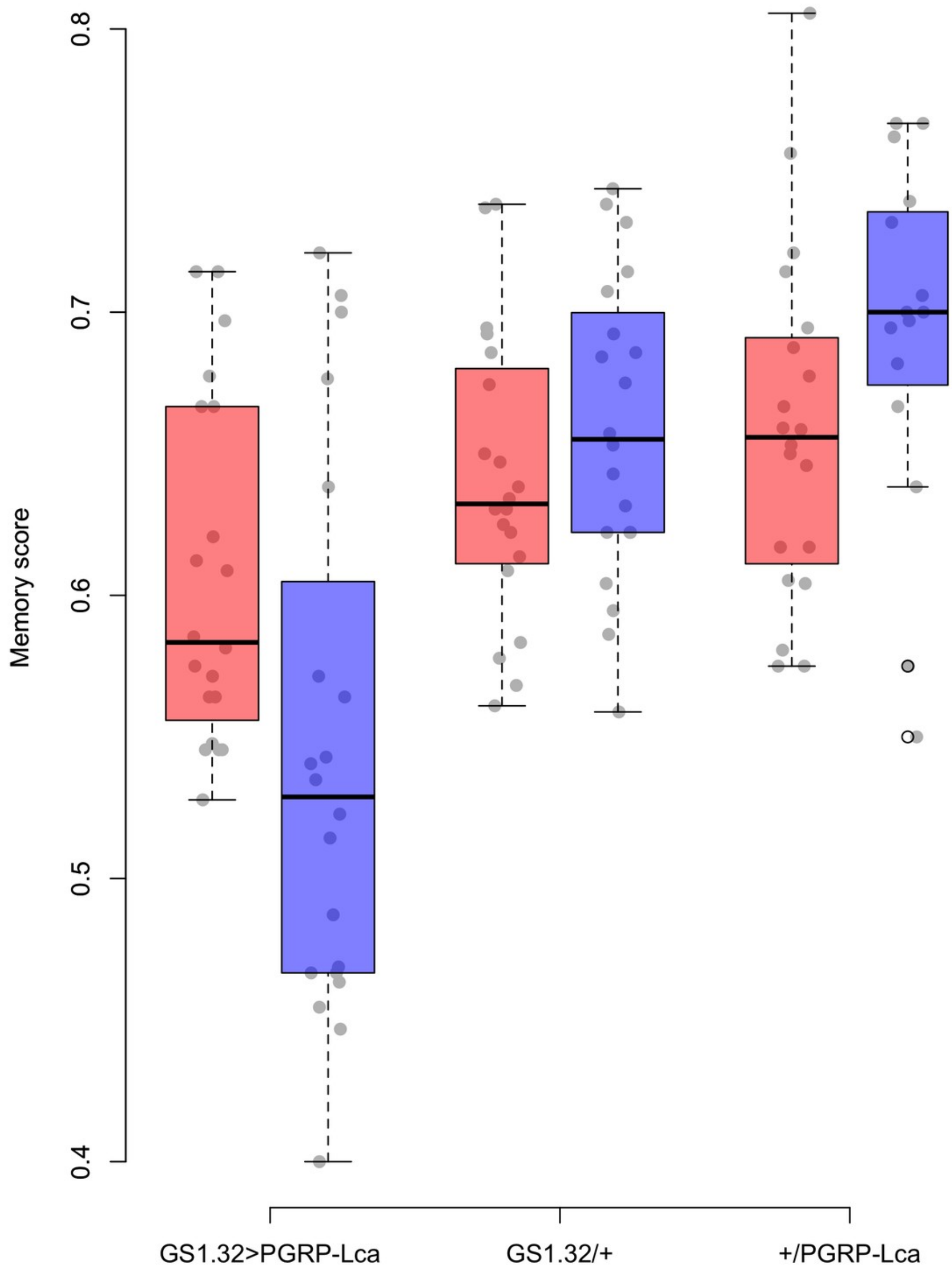




\section{Figure 2}

\section{Male sleepbins}

Fig 2. Sleepbins for the males for each genotype. The blue points represent the means for the RU486- flies. The red points represent the means of RU486+ flies. Error bars are standard errors. The shaded times are night (lights off).
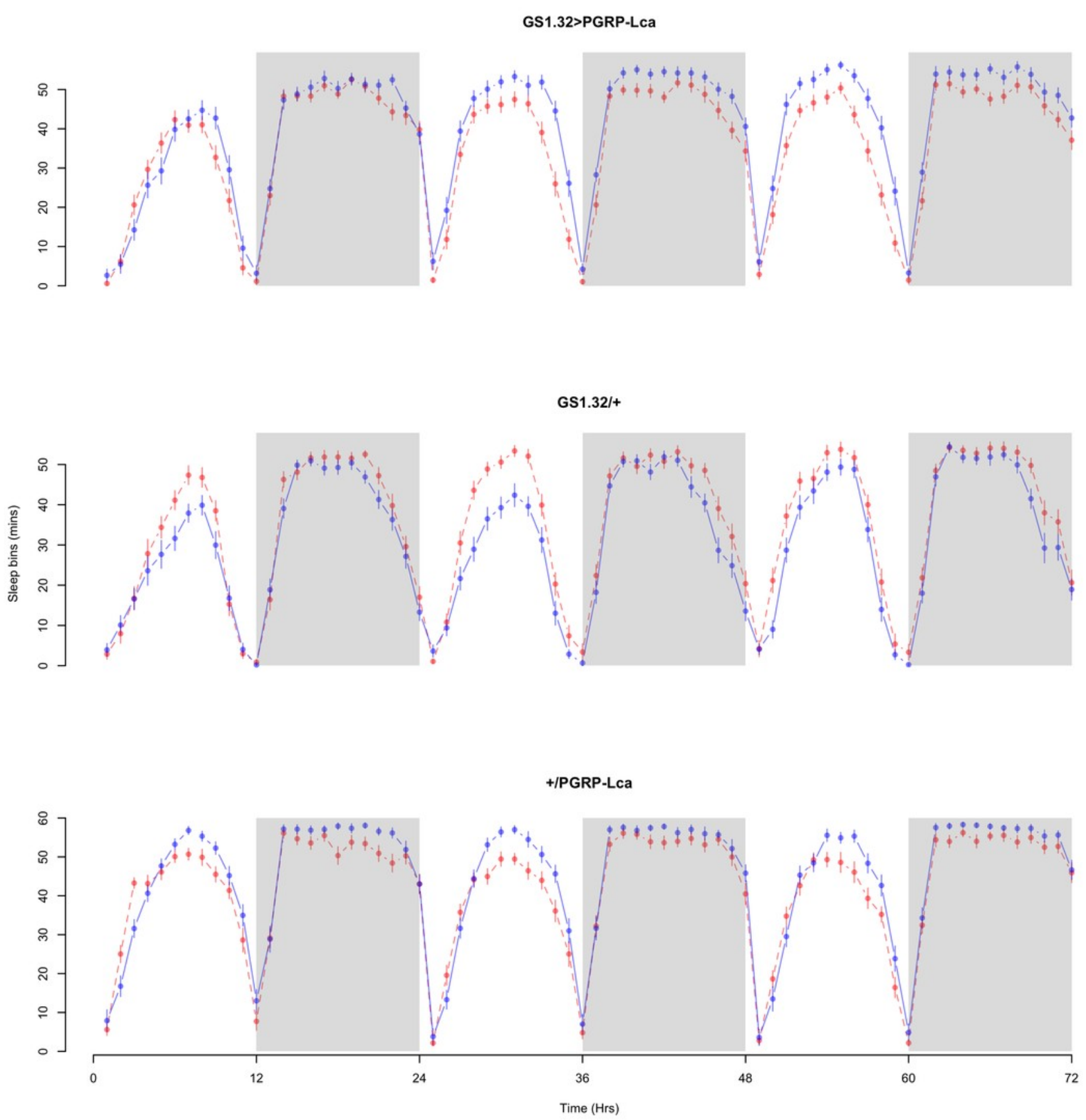


\section{Figure 3}

\section{Female sleepbins}

Fig 3. Sleepbins for the females for each genotype. The blue points represent the means for the RU486- flies. The red points represent the means of RU486+ flies. Error bars are standard errors. The shaded times are night (lights off).

GS1.32>PGRP-Lca

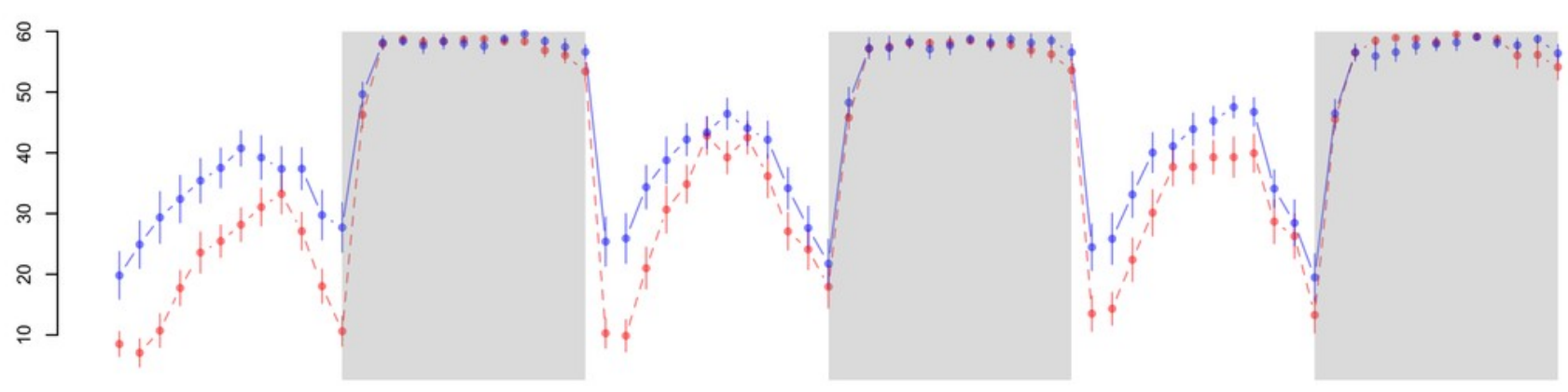

GS1.32/+

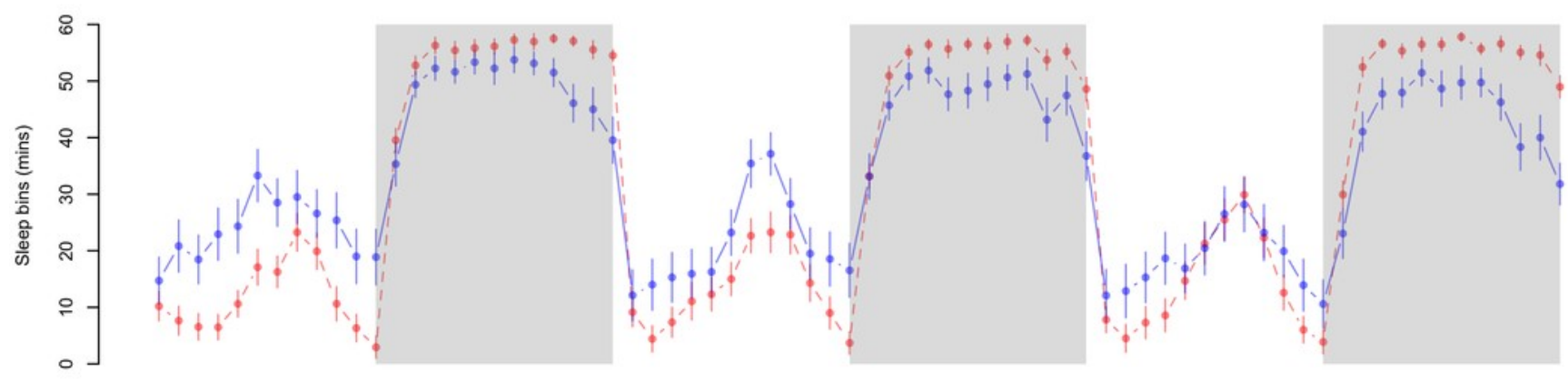

+/PGRP-Lca

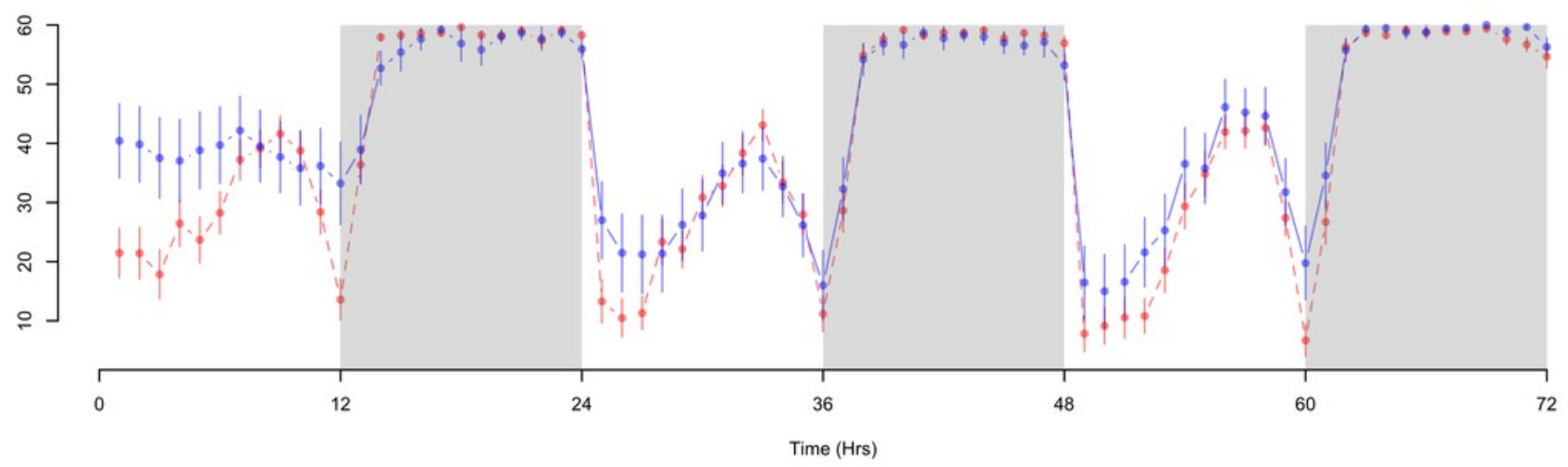




\section{Figure 4}

Male mean waking activity

Fig 4. Mean waking activity for the males for each genotype. The blue points represent the means for the RU486- flies. The red points represent the means of RU486+ flies. Error bars are standard errors. The shaded times are night (lights off).
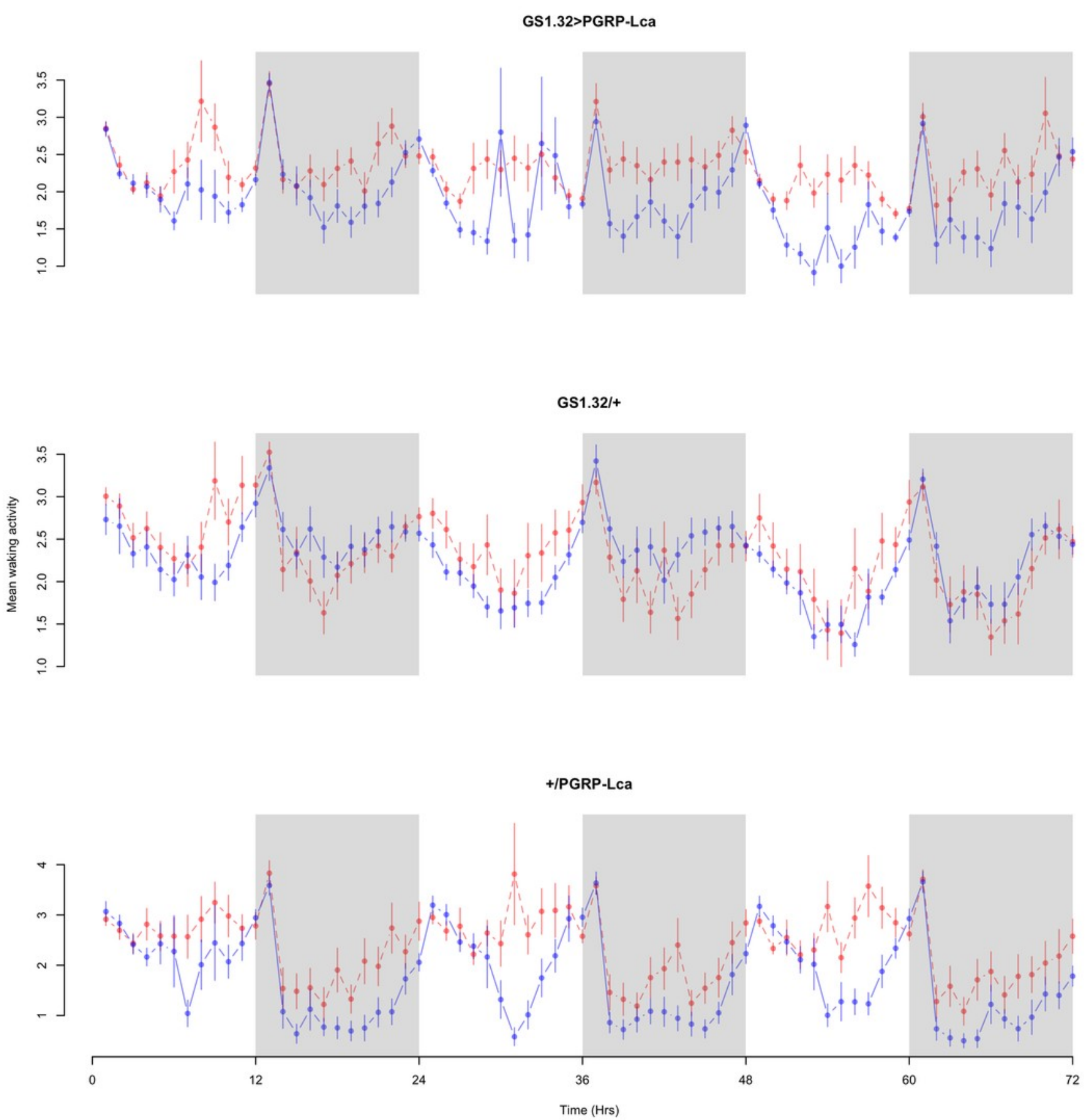


\section{Figure 5}

Female mean waking activity

Fig 5. Mean waking activity for the females for each genotype. The blue points represent the means for the RU486- flies. The red points represent the means of RU486+ flies. Error bars are standard errors. The shaded times are night (lights off).
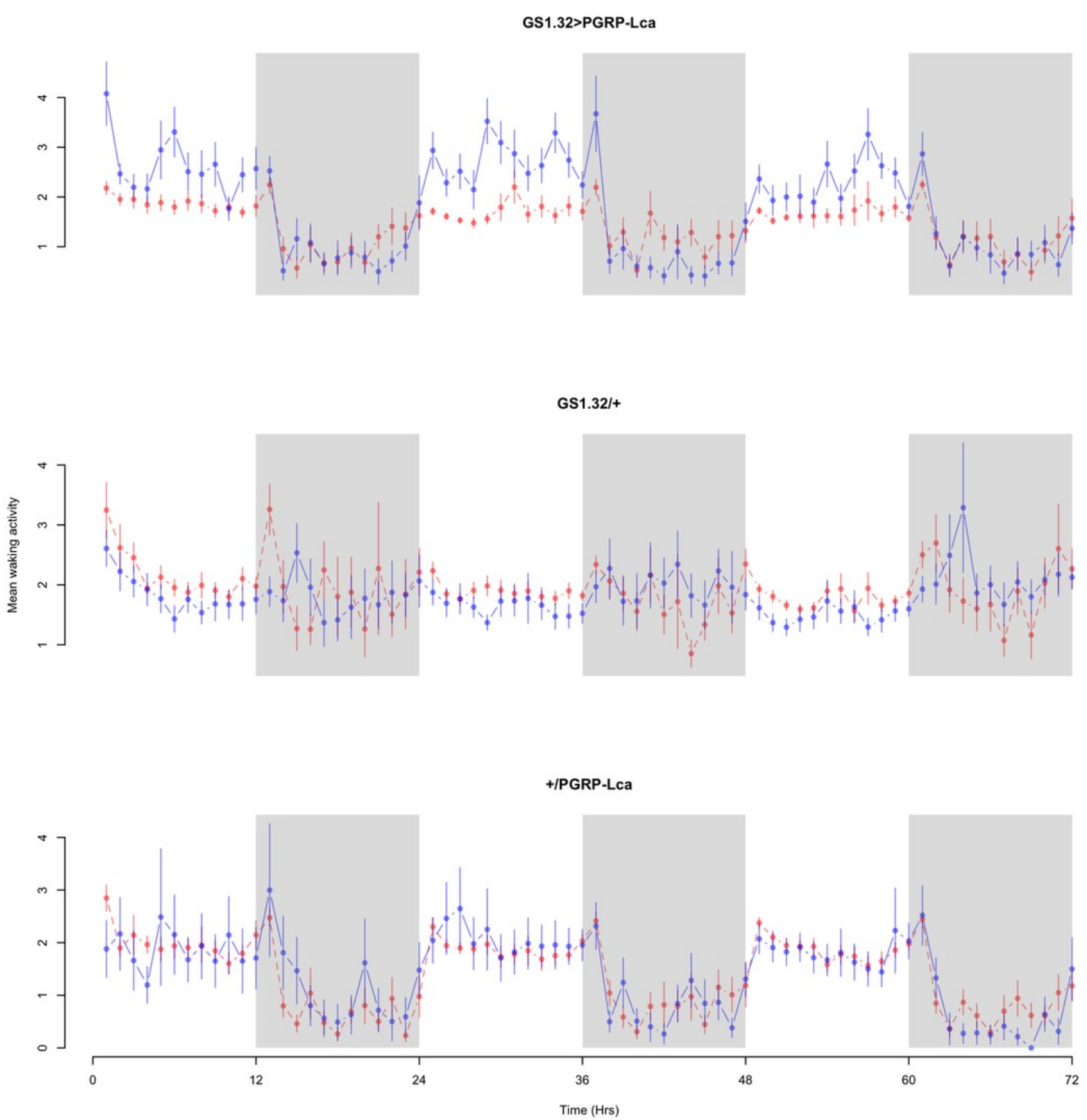


\section{Figure 6}

Male number of sleep bouts

Fig 6. Number of sleep bouts per hour for the males for each genotype. The blue points represent the means for the RU486- flies. The red points represent the means of RU486+ flies. Error bars are standard errors. The shaded times are night (lights off).
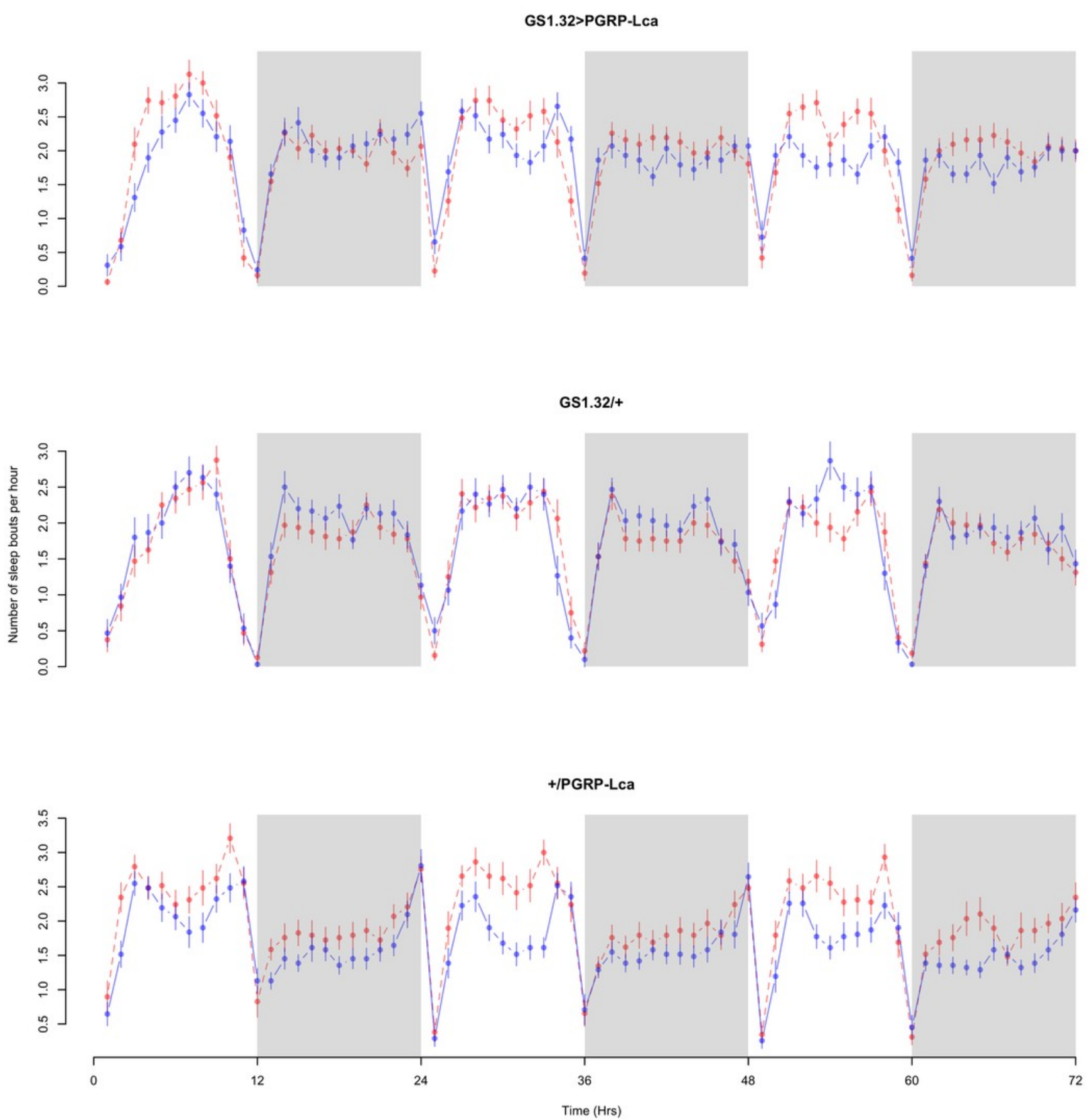


\section{Figure 7}

Female number of sleep bouts

Figure 7. Number of sleep bouts per hour for the females for each genotype. The blue points represent the means for the RU486- flies. The red points represent the means of RU486+ flies. Error bars are standard errors. The shaded times are night (lights off).
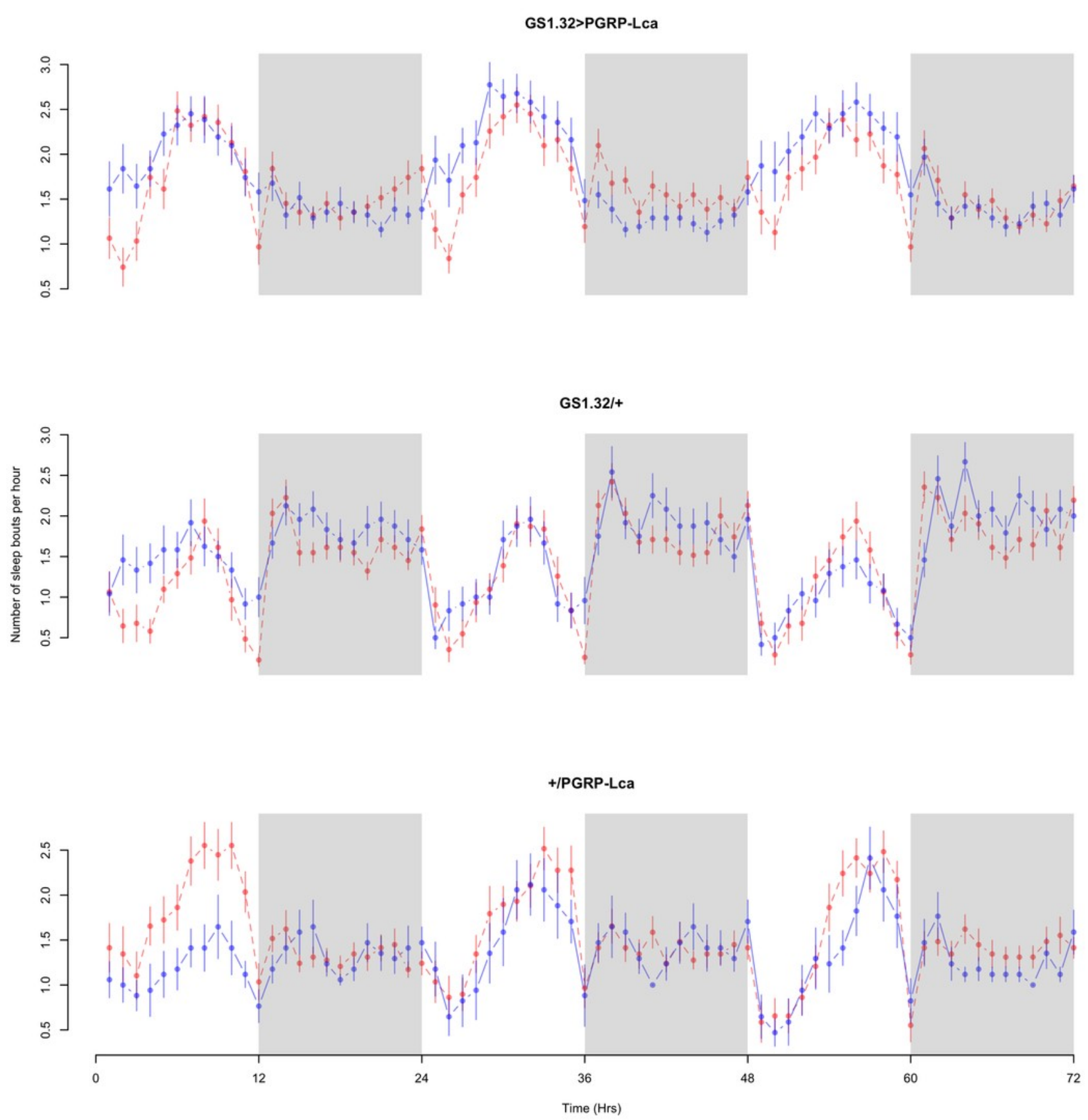


\section{Figure 8}

Male sleep bout duration

Fig 8. Sleep bout duration for the males for each genotype. The blue points represent the means for the RU486- flies. The red points represent the means of RU486+ flies. Error bars are standard errors. The shaded times are night (lights off).
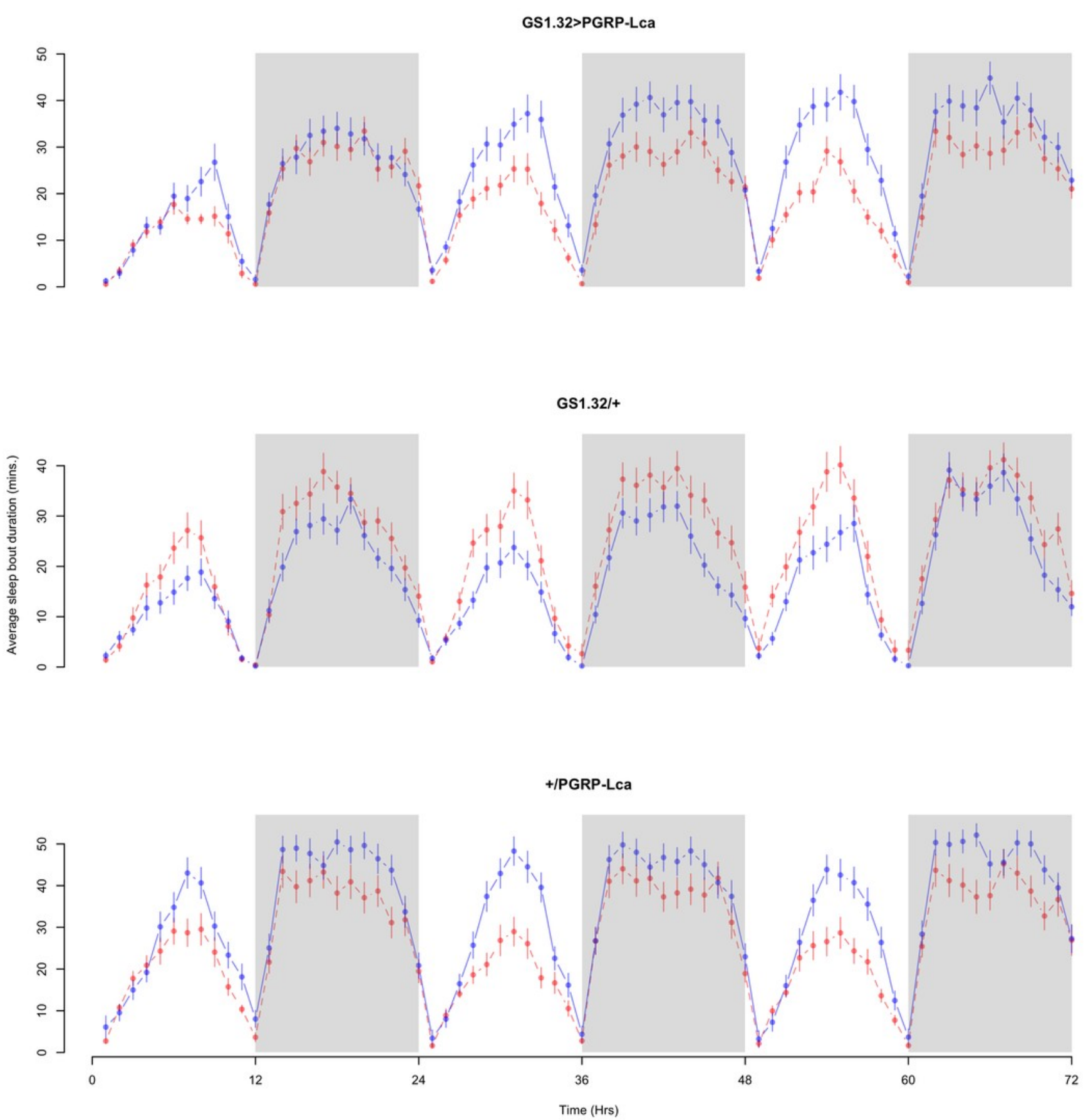


\section{Figure 9}

Female sleep bout duration

Fig 9. Sleep bout duration for the females for each genotype. The blue points represent the means for the RU486- flies. The red points represent the means of RU486+ flies. Error bars are standard errors. The shaded times are night (lights off).
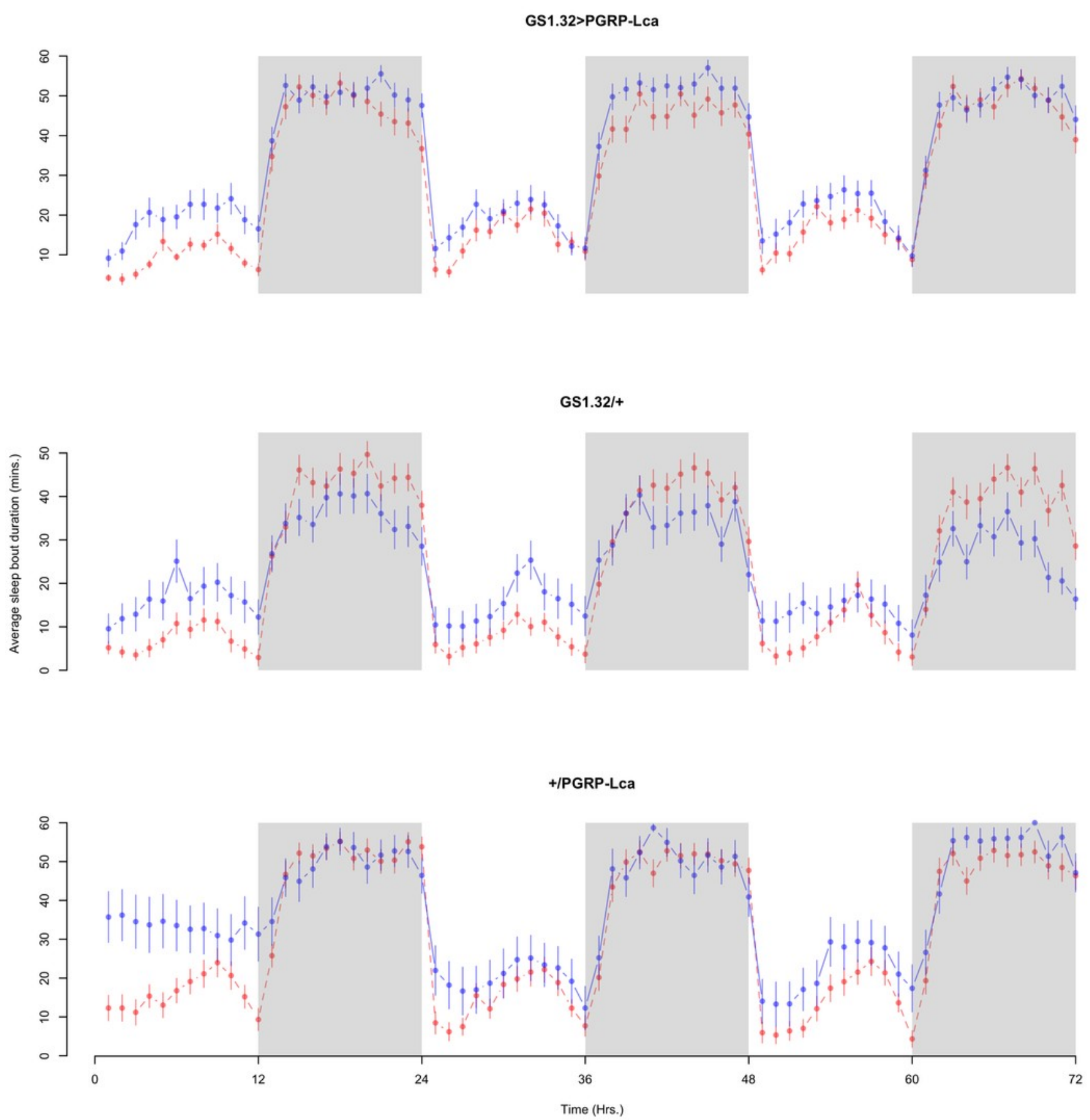


\section{Figure 10}

Male sleep latency

Fig 10. Sleep latency for the males for each genotype. The blue points represent the means for the RU486- flies. The red points represent the means of RU486+ flies. Error bars are standard errors.
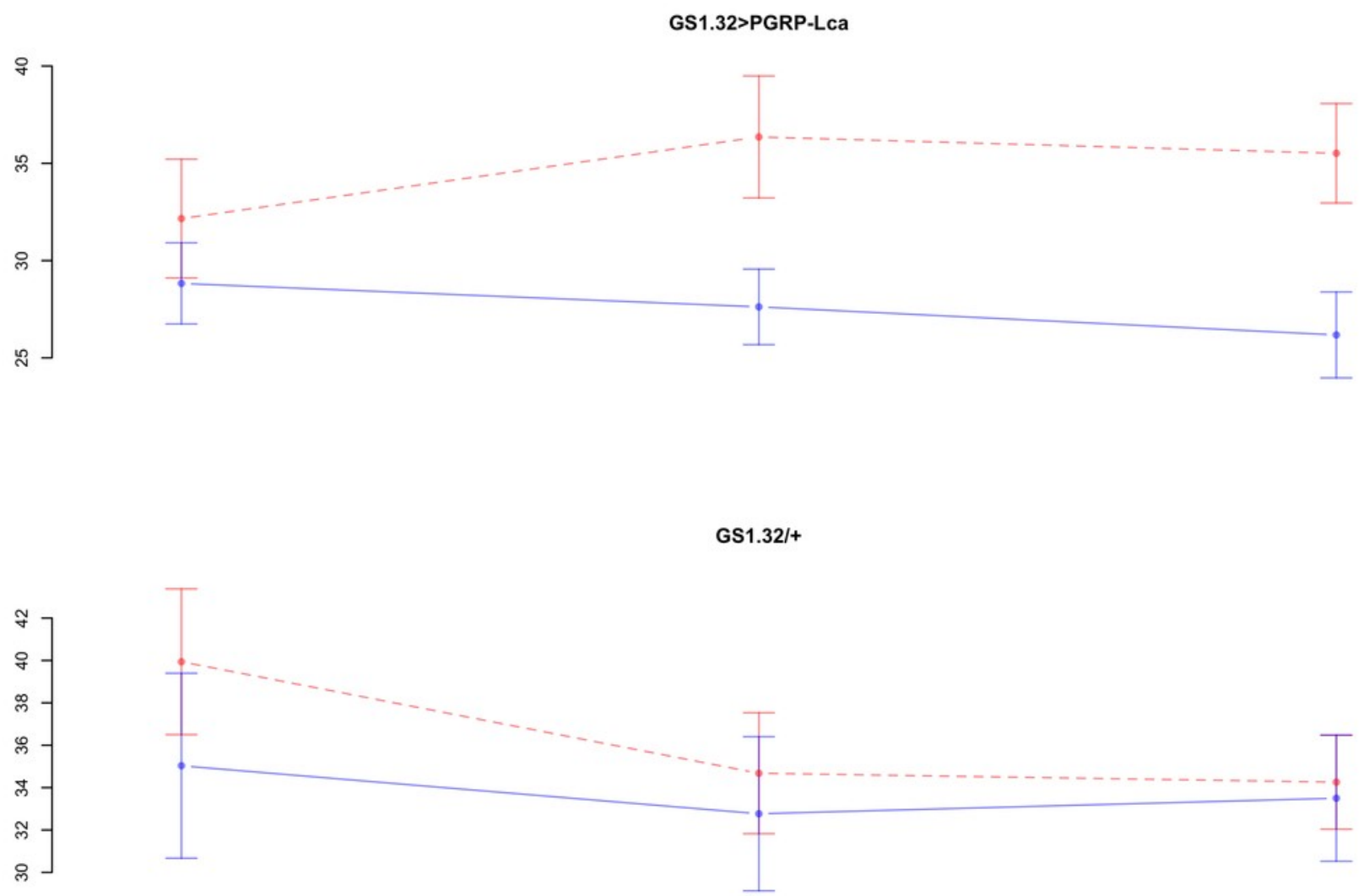

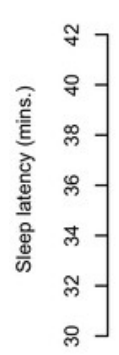

+/PGRP-Lca

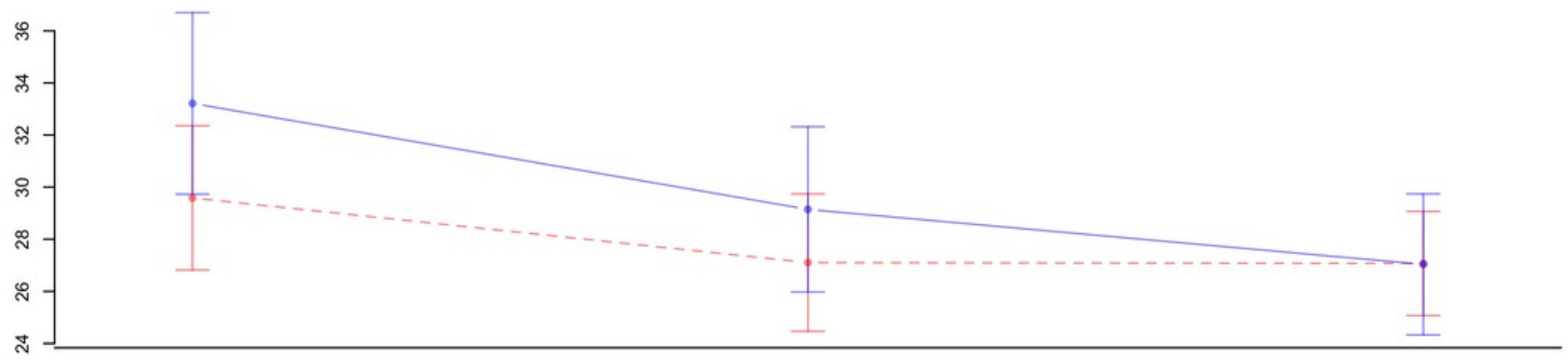

Time (Day) 


\section{Figure 11}

Female sleep latency

Fig 11. Sleep latency for the females for each genotype. The blue points represent the means for the RU486- flies. The red points represent the means of RU486+ flies. Error bars are standard errors.
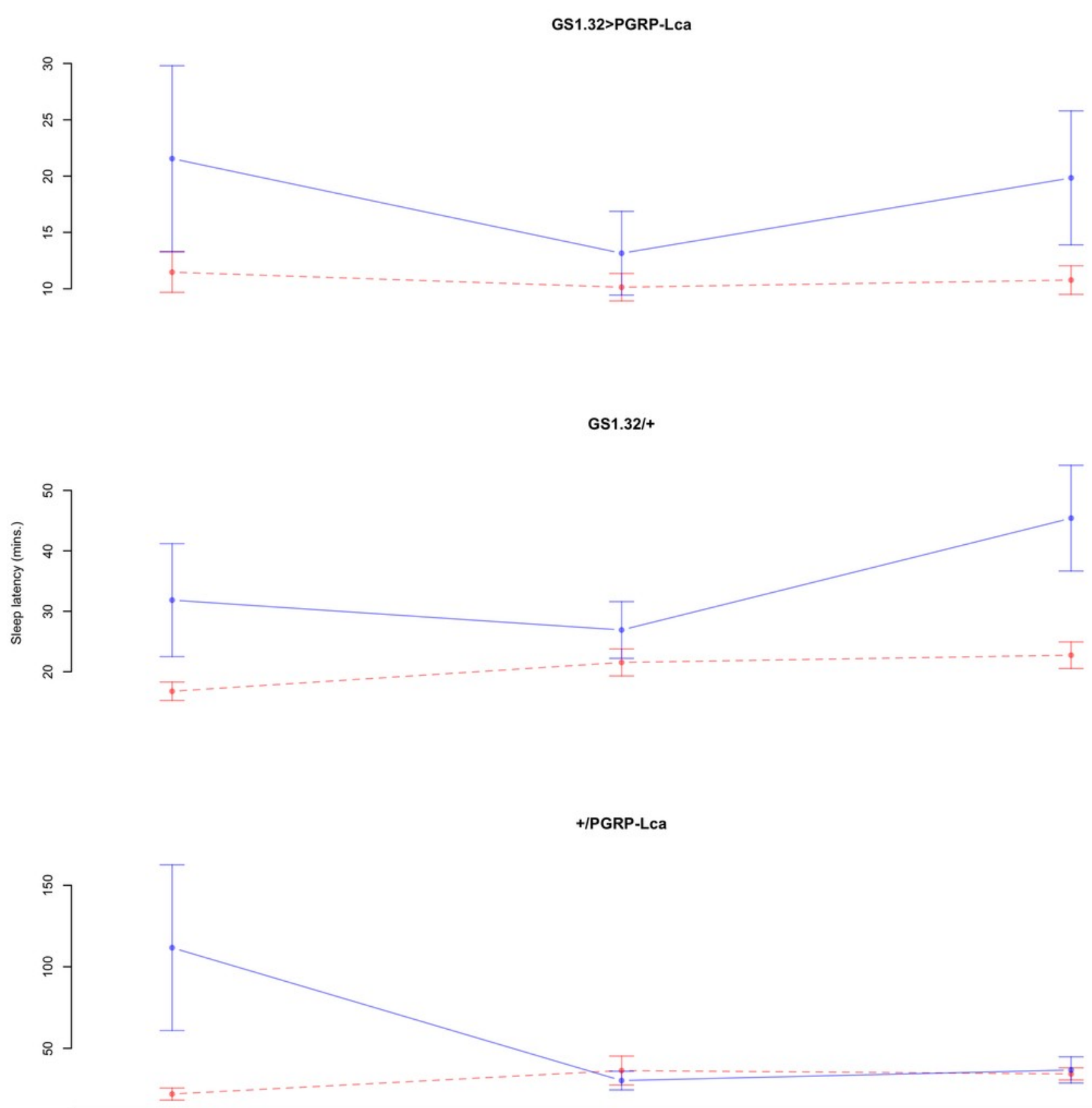

Time (Day) 


\section{Table 1 (on next page)}

Sleep bins

Table 1. Means and standard errors of the number of sleep bins per hour for the 12 groups of flies used in the sleep experiment. 


\begin{tabular}{llll}
\hline Male & & & \\
\hline Genotype & RU486- & RU486+ & $\%$ change \\
GS1.32>PGRP-Lca & $41.15+/-0.43$ & $36.41+/-0.41$ & $12 \%$ decrease \\
GS1.32/+ & $31.51+/-0.45$ & $35.89+/-0.45$ & $14 \%$ increase \\
+/ PGRP-Lca & $45.31+/-0.41$ & $42.45+/-0.40$ & $6 \%$ decrease \\
Female & & & \\
Genotype & RU486- & RU $486+$ & $10 \%$ decrease \\
GS1.32>PGRP-Lca & $45.84+/-0.41$ & $41.05+/-0.46$ & $1 \%$ decrease \\
\hline GS1.32/+ & $33.45+/-0.56$ & $33.11+/-0.53$ & $7 \%$ decrease \\
\hline +/ PGRP-Lca & $43.67+/-0.66$ & $40.67+/-0.50$ & \\
\hline
\end{tabular}




\section{Table 2 (on next page)}

Mean waking activity

Table 2. Means and standard errors of the number of times a fly crosses the beam per minute during 'waking' period (Mean waking activity) for the 12 groups of flies used in the sleep experiment. 


\begin{tabular}{llll}
\hline Male & & & \\
\hline Genotype & RU486- & RU486+ & $\%$ change \\
GS1.32>PGRP-Lca & $1.888+/-0.033$ & $2.324+/-0.027$ & $23 \%$ increase \\
GS1.32/+ & $2.252+/-0.024$ & $2.315+/-0.032$ & $3 \%$ increase \\
+/ PGRP-Lca & $1.725+/-0.039$ & $2.379+/-0.046$ & $38 \%$ increase \\
Female & & & \\
Genotype & RU486- & RU $486+$ & $\%$ change \\
GS1.32>PGRP-Lca & $1.790+/-0.046$ & $1.452+/-0.028$ & $19 \%$ decrease \\
GS1.32/+ & $1.817+/-0.042$ & $1.905+/-0.041$ & $5 \%$ increase \\
\hline +/ PGRP-Lca & $1.405+/-0.060$ & $1.398+/-0.031$ & $1 \%$ decrease \\
\hline
\end{tabular}




\section{Table 3 (on next page)}

Sleep bouts

Table 3. Means and standard errors of sleep bouts per hour for the 12 groups of flies used in the sleep experiment. 


\begin{tabular}{llll}
\hline Male & & & \\
\hline Genotype & RU486- & RU486+ & $\%$ change \\
GS1.32>PGRP-Lca & $1.849+/-0.024$ & $1.955+/-0.025$ & $6 \%$ increase \\
GS1.32/+ & $1.779+/-0.026$ & $1.695+/-0.025$ & $5 \%$ decrease \\
+/ PGRP-Lca & $1.673+/-0.023$ & $2.021+/-0.026$ & $21 \%$ increase \\
Female & & & \\
Genotype & RU486- & RU $486+$ & $\%$ change \\
GS1.32>PGRP-Lca & $1.755+/-0.024$ & $1.661+/-0.023$ & $5 \%$ decrease \\
GS1.32/+ & $1.555+/-0.029$ & $1.419+/-0.025$ & $9 \%$ decrease \\
\hline +/ PGRP-Lca & $1.324+/-0.030$ & $1.528+/-0.025$ & $15 \%$ increase \\
\hline
\end{tabular}




\section{Table 4 (on next page)}

Sleep bout duration

Table 4 Means and standard errors of sleep bout duration for the 12 groups of flies used in the sleep experiment. 


\begin{tabular}{llll}
\hline Male & & & \\
\hline Genotype & RU486- & RU486+ & $\%$ change \\
GS1.32>PGRP-Lca & $25.96+/-0.44$ & $20.05+/-0.34$ & $23 \%$ decrease \\
GS1.32/+ & $17.85+/-0.36$ & $23.27+/-0.42$ & $30 \%$ increase \\
+/ PGRP-Lca & $33.69+/-0.48$ & $26.74+/-0.45$ & $21 \%$ decrease \\
Female & & & \\
Genotype & RU486- & RU $486+$ & $\%$ change \\
GS1.32>PGRP-Lca & $34.17+/-0.49$ & $29.24+/-0.48$ & $14 \%$ decrease \\
GS1.32/+ & $23.21+/-0.54$ & $23.35+/-0.49$ & $1 \%$ increase \\
\hline +/ PGRP-Lca & $37.22+/-0.73$ & $31.29+/-0.54$ & $16 \%$ decrease \\
\hline
\end{tabular}




\section{Table 5 (on next page)}

Sleep latency

Table 5 Means and standard errors of sleep latency for the 12 groups of flies used in the sleep experiment. 


\begin{tabular}{llll}
\hline Male & & & \\
\hline Genotype & RU486- & RU486+ & $\%$ change \\
GS1.32>PGRP-Lca & $27.56+/-1.19$ & $34.68+/-1.68$ & $26 \%$ increase \\
GS1.32/+ & $33.77+/-2.11$ & $36.33+/-1.68$ & $8 \%$ increase \\
\hline +/ PGRP-Lca & $29.81+/-1.81$ & $27.93+/-1.43$ & $6 \%$ decrease \\
Female & & & \\
Genotype & RU486- & RU $486+$ & $\%$ change \\
GS1.32>PGRP-Lca & $18.22+/-3.67$ & $10.79+/-0.83$ & $41 \%$ decrease \\
\hline GS1.32/+ & $34.94+/-4.58$ & $20.34+/-1.18$ & $42 \%$ decrease \\
\hline +/ PGRP-Lca & $57.11+/-16.54$ & $31.04+/-3.48$ & $46 \%$ decrease \\
\hline
\end{tabular}

